\title{
Correction to: Relativistic quantum chemistry involving heavy atoms
}

\author{
Matteo De Santis ${ }^{1} \cdot$ Leonardo Belpassi $^{2}$ (D) Francesco Tarantelli $^{1} \cdot$ Loriano Storchi $^{3}$
}

Published online: 6 October 2018

(c) Accademia Nazionale dei Lincei 2018

\section{Correction to: Rendiconti Lincei. Scienze Fisiche e Naturali (2018) 29:209-217 \\ https://doi.org/10.1007/s12210-018-0706-7}

In the original publication, second author's name was incorrectly published as 'Leonardo Belapassi'. The correct name should read as 'Leonardo Belpassi'.

The original article can be found online at https://doi.org/10.1007/ s12210-018-0706-7.

Leonardo Belpassi

leonardo.belpassi@cnr.it

Francesco Tarantelli

francesco.tarantelli@unipg.it

Loriano Storchi

loriano@storchi.org

1 Dipartimento di Chimica, Biologia e Biotecnologie,

Università degli Studi di Perugia, Via Elce di Sotto 8,

06123 Perugia, Italy

2 Istituto di Scienze e Tecnologie Molecolari, Consiglio

Nazionale delle Ricerche c/o Dipartimento di Chimica,

Biologia e Biotecnologie, Università degli Studi di Perugia,

Via Elce di Sotto 8, 06123 Perugia, Italy

3 Istituto di Scienze e Tecnologie Molecolari, Consiglio

Nazionale delle Ricerche c/o Dipartimento di Farmacia,

Università degli Studi 'G. D’Annunzio', Via dei Vestini 31,

66100 Chieti, Italy 